\title{
Oblique corrections in the Dine-Fischler-Srednicki axion model
}

\author{
Alisa Katanaeva ${ }^{1, a}$ and Domènec Espriu ${ }^{2}$ \\ ${ }^{1}$ V. A. Fock Department of Theoretical Physics, Saint-Petersburg State University, 1 ul. Ulyanovskaya, \\ 198504, Russia \\ ${ }^{2}$ Departament d'Estructura i Constituents de la Matèria, Institut de Ciències del Cosmos (ICCUB), Universi- \\ tat de Barcelona, Martí Franquès 1, 08028 Barcelona, Spain
}

\begin{abstract}
In the Minimal Standard Model (MSM) there is no degree of freedom for dark matter. There are several extensions of the MSM introducing a new particle - an invisible axion, which can be regarded as a trustworthy candidate at least for a part of the dark matter component. However, as it is extremely weakly coupled, it cannot be directly measured at the LHC. We propose to explore the electroweak sector indirectly by considering a particular model that includes the axion and derive consequences that could be experimentally tested.

We discuss the Dine-Fischler-Srednicki (DFS) model, which extends the two-Higgs doublet model with an additional Peccei-Quinn symmetry and leads to a physically acceptable axion. The non-linear parametrization of the DFS model is exploited in the generic case where all scalars except the lightest Higgs and the axion have masses at or beyond the $\mathrm{TeV}$ scale. We compute the oblique corrections and use their values from the electroweak experimental fits to put constraints on the mass spectrum of the DFS model.
\end{abstract}

\section{Introduction}

In this paper we reexamine the Dine-Fischler-Srednicki-Zhitnitsky (DFSZ) model. It is the twoHiggs doublet model (2HDM) containing an additional singlet, endowed with a Peccei-Quinn (PQ) symmetry. Introduction of the PQ symmetry in the Standard Model (SM) leads to the solution of the strong CP problem but induces the necessity of two Higgs doublets and the presence of an axion. The last becomes physically acceptable in the DFSZ model. From one point of view, the model includes a lot of new physics coming from the 2HDM. From another, an invisible axion is a possible candidate at least for a part of the dark matter. Both these reasons, as well as the interplay between the 2HDM content and the axion, make this model interesting to study.

The discovery of a Higgs-like particle with $m_{h} \sim 126 \mathrm{GeV}$ and the development of experiments, now probing the predictions of the standard electroweak theory with sufficient accuracy, impose the constraints on any potential new physics that might exist at higher energies. Nevertheless, there is still a room for a wide variety of the $2 \mathrm{HDM}$ scenarios. In the same time the introduction of an axion restricts the number of possibilities, making the phenomenological consequences of the DFSZ model more rigorous.

\footnotetext{
a e-mail: alice.katanaeva@gmail.com
} 


\section{Fields and symmetries}

The DFSZ model originated as a solution to several problems. Firstly, Peccei and Quinn [1] proposed to introduce an additional global $U(1)_{P Q}$ symmetry to the SM to rotate away the CP violating term. Then, the introduction of two Higgs doublets is necessary to make the SM invariant under the $U(1)_{P Q}$ transformation. The last thing is to make a new Goldstone boson of the necessarily broken PQ symmetry - an axion - invisible, meaning it should have a very light mass and be very weakly coupled to ordinary matter. It wasn't achieved in the original PQ model. However, later Dine, Fischler and Srednicki [2] and Zhitnitsky [3] independently proposed a generalization of the Peccei-Quinn scheme with a harmless axion. Addition of a complex scalar field gives an axion the right properties.

So, we work with two Higgs doublets and a complex scalar singlet:

$$
\phi_{1}=\left(\begin{array}{c}
\alpha_{+} \\
\alpha_{0}
\end{array}\right) ; \quad \phi_{2}=\left(\begin{array}{c}
\beta_{+} \\
\beta_{0}
\end{array}\right) ; \quad \phi .
$$

The vevs of the fields are

$$
\left\langle\phi_{1}\right\rangle=\left(\begin{array}{c}
0 \\
v_{1}
\end{array}\right) ; \quad\left\langle\phi_{2}\right\rangle=\left(\begin{array}{c}
0 \\
v_{2}
\end{array}\right) ;\langle\phi\rangle=v_{\phi} .
$$

We can define the usual electroweak vacuum expectation value as $v^{2}=\left(v_{1}^{2}+v_{2}^{2}\right) / 2=246 \mathrm{GeV}$ and the well-known $2 \mathrm{HDM}$ parameter $\tan \beta=v_{2} / v_{1}$. The DFSZ model includes a $2 \mathrm{HDM}$ of type II: $\phi_{1}$ couples only to right-handed charge $2 / 3$ quarks, $\phi_{2}$ couples only to the right-handed charge $-1 / 3$ quarks and to right-handed charged leptons. The Yukawa terms have the structure:

$$
\mathcal{L}_{Y}=G_{1} \bar{q}_{L} \tilde{\phi}_{1} u_{R}+G_{2} \bar{q}_{L} \phi_{2} d_{R}+G_{3} \bar{l}_{L} \phi_{2} e_{R}+\text { h.c. },
$$

and similarly for other quarks and leptons. Here $\tilde{\phi}_{i}=i \tau_{2} \phi_{i}^{*}$.

For the potential we choose the one respecting $\mathrm{CP}, S U(2) \times U(1)$ and $U(1)_{P Q}$ symmetries:

$$
\begin{aligned}
& V\left(\phi, \phi_{1}, \phi_{2}\right)=\lambda_{\phi}\left(\phi^{*} \phi-V_{\phi}^{2}\right)^{2}+\lambda_{1}\left(\phi_{1}^{\dagger} \phi_{1}-V_{1}^{2}\right)^{2}+\lambda_{2}\left(\phi_{2}^{\dagger} \phi_{2}-V_{2}^{2}\right)^{2}+\lambda_{3}\left(\phi_{1}^{\dagger} \phi_{1}-V_{1}^{2}+\phi_{2}^{\dagger} \phi_{2}-V_{2}^{2}\right)^{2}+ \\
& +\lambda_{4}\left[\left(\phi_{1}^{\dagger} \phi_{1}\right)\left(\phi_{2}^{\dagger} \phi_{2}\right)-\left(\phi_{1}^{\dagger} \phi_{2}\right)\left(\phi_{2}^{\dagger} \phi_{1}\right)\right]+\left(a \phi_{1}^{\dagger} \phi_{1}+b \phi_{2}^{\dagger} \phi_{2}\right) \phi^{*} \phi+c\left(\phi_{1}^{\dagger} \phi_{2} \phi^{2}+\phi_{2}^{\dagger} \phi_{1} \phi^{* 2}\right) .
\end{aligned}
$$

Furthermore, we consider an additional possible symmetry - the custodial symmetry. It is an approximate symmetry in the SM keeping the equality $\rho \equiv \frac{M_{W}^{2}}{M_{Z}^{2} \cos ^{2} \theta_{W}} \simeq 1$ even when $g^{\prime} \neq 0$, with $\theta_{W}$ being the Weinberg angle and $M_{W}, M_{Z}$ - the electroweak gauge boson masses.

To make more evident such global symmetries of the potential of the DFSZ model one can introduce a matrix notation [4]. Firstly, we construct the $2 \times 2$ matrices from the fields of Higgs doublets:

$$
\Phi_{12}=\left(\tilde{\phi}_{1} \phi_{2}\right)=\left(\begin{array}{cc}
\alpha_{0}^{*} & \beta_{+} \\
-\alpha_{-} & \beta_{0}
\end{array}\right), \quad \Phi_{21}=\left(\tilde{\phi}_{2} \phi_{1}\right)=\left(\begin{array}{cc}
\beta_{0}^{*} & \alpha_{+} \\
-\beta_{-} & \alpha_{0}
\end{array}\right)=\tau_{2} \Phi_{12}^{*} \tau_{2} .
$$

Secondly, we consider the following combinations:

$$
I=\Phi_{12}^{\dagger} \Phi_{12}=\left(\begin{array}{cc}
\phi_{1}^{\dagger} \phi_{1} & \tilde{\phi}_{1}^{\dagger} \phi_{2} \\
-\phi_{1}^{\dagger} \tilde{\phi}_{2} & \phi_{2}^{\dagger} \phi_{2}
\end{array}\right), \quad J=\Phi_{12}^{\dagger} \Phi_{21}=\left(\begin{array}{cc}
\phi_{2}^{\dagger} \phi_{1} & 0 \\
0 & \phi_{2}^{\dagger} \phi_{1}
\end{array}\right) .
$$

Their vevs are

$$
\langle I\rangle=\left(\begin{array}{cc}
v_{1}^{2} & 0 \\
0 & v_{2}^{2}
\end{array}\right), \quad\langle J\rangle=v_{1} v_{2}
$$


This vacuum is not invariant under the full group $S U(2)_{L} \times S U(2)_{R}$. However, if $v_{1}=v_{2}$, then $\langle I\rangle$ is proportional to the $2 \times 2$ identity matrix and the vacuum preserves a group $S U(2)_{V}$ (the $V$ stands for "vectorial"), corresponding to identical matrices, i.e. $L=R$. This remaining group preserved by the vacuum is the custodial-symmetry group.

The last thing is to define the constant matrix $W$,

$$
W=\left(V_{1}^{2}+V_{2}^{2}\right) \frac{\mathbf{I}}{2}+\left(V_{1}^{2}-V_{2}^{2}\right) \frac{\tau_{3}}{2}=\left(\begin{array}{cc}
V_{1}^{2} & 0 \\
0 & V_{2}^{2}
\end{array}\right),
$$

and then, the potential can be written as:

$$
\begin{aligned}
& V(\phi, I, J)=\lambda_{\phi}\left(\phi^{*} \phi-V_{\phi}^{2}\right)^{2}+\frac{\lambda_{1}}{4}\left\{\operatorname{Tr}\left[(I-W)\left(1+\tau_{3}\right)\right]\right\}^{2}++\frac{\lambda_{2}}{4}\left\{\operatorname{Tr}\left[(I-W)\left(1-\tau_{3}\right)\right]\right\}^{2}+ \\
& +\lambda_{3}[\operatorname{Tr}(I-W)]^{2}+\frac{\lambda_{4}}{4} \operatorname{Tr}\left[I^{2}-\left(I \tau_{3}\right)^{2}\right]+\frac{1}{2} \operatorname{Tr}\left[(a+b) I+(a-b) I \tau_{3}\right] \phi^{*} \phi+\frac{c}{2} \operatorname{Tr}\left(J \phi^{2}+J^{\dagger} \phi^{* 2}\right) .
\end{aligned}
$$

A custodial global $S U(2)_{L} \times S U(2)_{R}$ transformation acts on our fields as

$$
\Phi_{i j} \rightarrow L \Phi_{i j} R^{\dagger}, \quad I \rightarrow R I R^{\dagger}, \quad J \rightarrow J .
$$

If $S U(2)_{L} \times S U(2)_{R}$ is to be a symmetry, the parameters of the potential have to be set according to the custodial relations: $\lambda_{1}=\lambda+\lambda_{B}, \lambda_{2}=\lambda-\lambda_{B}, \lambda_{4}=2 \lambda+\lambda_{4 B}, V_{1}^{2}=V^{2}+V_{B}^{2}, V_{2}^{2}=V^{2}-V_{B}^{2}$, $a+a_{B}=b$. All the "B" parameters vanish in the limit of custodial symmetry. In total, there are 11 parameters of which 7 are custodial preserving and 4 are custodial breaking.

\section{Effective potential}

In this section we integrate out the heavy scalars in order to build a low-energy effective theory at the $\mathrm{TeV}$ scale with an axion and a light Higgs.

We decompose the matrix-valued $\Phi_{12}$ field in the following form $\Phi_{12}=\mathcal{U} \mathcal{M}_{12} \cdot \mathcal{U}=\exp \left(i \frac{\vec{G} \cdot \vec{t}}{v}\right)$ is a $2 \times 2$ matrix containing the three Goldstone bosons $G_{i}$ associated to the breaking of $S U(2)_{L} \times U(1)_{Y}$ to $U(1)_{e m}$. Note that the matrices $I$ and $J$ entering the DFSZ potential are actually independent of $\mathcal{U}$. The effective potential then does depend only on the degrees of freedom contained in $\mathcal{M}_{12}$.

There is also the singlet field in the scalar potential, it can be parametrized as: $\phi=\rho+i G_{\phi}$. The phase of $\phi$ does not drop from the potential automatically because of the $c$ term in (4). $G_{\phi}$ mixes with the usual $0^{-}$scalar from the $2 \mathrm{HDM}$. To have a well-defined massless state we need to find a suitable phase both in $\mathcal{M}_{12}$ and in $\phi$ that drops from the potential.

It is straightforward to define the phases from the requirement that the kinetic terms are diagonal and exhibit the canonical normalization. Then, the non-linear parametrization of $\Phi_{12}$ reads as ${ }^{1}$

$$
\Phi_{12}=\mathcal{U} M_{12} U_{a}
$$

with a unitary matrix containing the axion being $U_{a}=\exp \left(i \frac{2 a_{\phi}}{\sqrt{v_{\phi}^{2}+v^{2} s_{2 \beta}^{2}}}\left(\begin{array}{cc}s_{\beta}^{2} & 0 \\ 0 & c_{\beta}^{2}\end{array}\right)\right)$ and the heavy scalars collected in the matrix

$$
M_{12}=\sqrt{2}\left(\begin{array}{ll}
(v+H) c_{\beta}-\left(S-\frac{i v_{\phi}}{\sqrt{v_{\phi}^{2}+v^{2} s_{2 \beta}^{2}}} \tilde{A}_{0}\right) s_{\beta} & \sqrt{2} H_{+} c_{\beta} \\
\sqrt{2} H_{-} s_{\beta} & (v+H) s_{\beta}+\left(S+\frac{i v_{\phi}}{\sqrt{v_{\phi}^{2}+v^{2} s_{2 \beta}^{2}}} \tilde{A_{0}}\right) c_{\beta}
\end{array}\right) .
$$

\footnotetext{
${ }^{1}$ Here we introduce the short-hand notation $s_{m \beta}^{n} \equiv \sin ^{n}(m \beta)$ and $c_{m \beta}^{n} \equiv \cos ^{n}(m \beta)$.
} 
The field redefinition we use is as follows:

$$
v+H=\frac{c_{\beta}}{\sqrt{2}} \mathfrak{R}\left[\alpha_{0}\right]+\frac{s_{\beta}}{\sqrt{2}} \mathfrak{R}\left[\beta_{0}\right], \quad S=-\frac{s_{\beta}}{\sqrt{2}} \mathfrak{R}\left[\alpha_{0}\right]+\frac{c_{\beta}}{\sqrt{2}} \mathfrak{R}\left[\beta_{0}\right], \quad H_{ \pm}=\frac{c_{\beta} \beta_{ \pm}-s_{\beta} \alpha_{ \pm}}{2} .
$$

The singlet field is non-linearly parametrized as

$$
\phi=\left(v_{\phi}+\rho-i \frac{v s_{2 \beta}}{\sqrt{v_{\phi}^{2}+v^{2} s_{2 \beta}^{2}}} \tilde{A}_{0}\right) \exp \left(i \frac{a_{\phi}}{\sqrt{v_{\phi}^{2}+v^{2} s_{2 \beta}^{2}}}\right) .
$$

The fields of $H, S$ and $\rho$ are not mass eigenstates but they have vanishing vevs. The mass eigenstates are defined through the rotation matrix $R$ :

$$
H=\sum_{i=1}^{3} R_{H i} h_{i}, \quad S=\sum_{i=1}^{3} R_{S i} h_{i}, \quad \rho=\sum_{i=1}^{3} R_{\rho i} h_{i} .
$$

$H, S$ are also called interaction eigenstates. For instance, $H$ has the same coupling to the gauge fields as the SM Higgs boson, but the Higgs mass $m_{h} \simeq 126 \mathrm{GeV}$ is attributed to the lightest of $h_{i}$ states.

The construction of the effective Lagrangian for the DFSZ model goes with inclusion of additional light particles explicitly as dynamical states:

$$
\begin{aligned}
& \mathcal{L}=\frac{v^{2}}{4}\left(1+2 g_{1} \frac{h}{v}+g_{2} \frac{h^{2}}{v^{2}}+\ldots\right) \operatorname{Tr} \mathcal{D}_{\mu} \mathcal{U}^{\dagger} \mathcal{D}^{\mu} \mathcal{U}+\left(\frac{v_{\phi}^{2}}{v_{\phi}^{2}+v^{2} \sin ^{2} 2 \beta}\right) \partial_{\mu} a_{\phi} \partial^{\mu} a_{\phi}+\frac{1}{2} \partial_{\mu} h \partial^{\mu} h- \\
& -V(h)+\sum_{i=0}^{13} a_{i}\left(\frac{h}{v}\right) O_{i}+\mathcal{L}_{r e n},
\end{aligned}
$$

where the $O_{i}$ is a set of local gauge invariant operators [4],

$$
\begin{aligned}
& \mathcal{D}_{\mu} \mathcal{U}=D_{\mu} \mathcal{U}+\mathcal{U}\left(\partial_{\mu} U_{a}\right) U_{a}^{\dagger}, \quad V(h)=\frac{m_{h}^{2}}{2} h^{2}-d_{3}(\lambda v) h^{3}-d_{4} \frac{\lambda}{4} h^{4}, \\
& \mathcal{L}_{r e n}=\frac{c_{1}}{v^{4}}\left(\partial_{\mu} h \partial^{\mu} h\right)^{2}+\frac{c_{2}}{v^{2}} \operatorname{Tr} \mathcal{D}_{\mu} \mathcal{U}^{\dagger} \mathcal{D}^{\mu} \mathcal{U}+\frac{c_{3}}{v^{2}}\left(\partial_{\mu} h \partial^{v} h\right) \operatorname{Tr} \mathcal{D}^{\mu} \mathcal{U}^{\dagger} \mathcal{D}_{\nu} \mathcal{U} .
\end{aligned}
$$

The terms in $\mathcal{L}_{\text {ren }}$ are required for renormalizability [5] at the one-loop level and play no role in the discussion. The couplings $a_{i}$ are functions of $h / v$ and can be regularly expanded. Their constant parts $a_{i}(0)$ are related to the electroweak precision parameters.

\section{Mass eigenstates}

Having defined the fields we proceed with the description of the mass spectrum of the model (following [6]). We have two doublets and a singlet, so a total of $4+4+2=10$ spin-zero particles. Three particles are eaten by the gauge bosons and 7 scalars fields are left in the spectrum: two charged Higgs bosons, two $0^{-}$states and three neutral $0^{+}$states.

The charged Higgs bosons have a mass $m_{H_{ \pm}}^{2}=8\left(\lambda_{4} v^{2}+\frac{c v_{\phi}^{2}}{s_{2 \beta}}\right)$. In the $0^{-}$sector $A_{0}$ and $G_{\phi}$ fields mix forming the massless state, the axion $a_{\phi}=\frac{v s_{2 \beta} A_{0}+v_{\phi} G_{\phi}}{\sqrt{v_{\phi}^{2}+v^{2} s_{2 \beta}^{2}}}$, and the massive state $\tilde{A}_{0}=\frac{v_{\phi} A_{0}-v s_{2 \beta} G_{\phi}}{\sqrt{v_{\phi}^{2}+v^{2} s_{2 \beta}^{2}}}$, with $m_{\tilde{A}_{0}}^{2}=8 c\left(\frac{v_{\phi}^{2}}{s_{2 \beta}}+v^{2} s_{2 \beta}\right)$. 


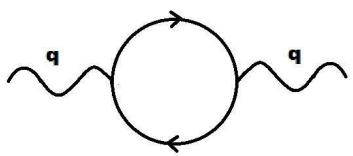

(a)

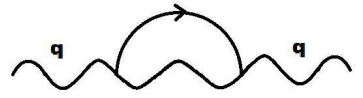

(b)

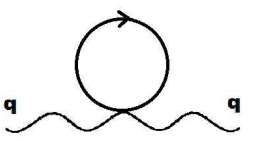

(c)

Figure 1. Types of Feynman diagrams occurring in the calculation of the vacuum polarizations.

As was mentioned before, there is mixing in the $0^{+}$sector. We mark the corresponding $0^{+}$mass eigenstates as $h_{i}$. The mass matrix can be diagonalized in the limit of large $v_{\phi}$, which is astrophysically constrained to be at least of order $10^{7} \mathrm{GeV}$. In the paper [6] an expansion in $v / v_{\phi}$ is carried out to the second order to get the masses of $h_{i}$. It is argued that the nominal expansion in powers of $v / v_{\phi}$ is applicable in a number of cases.

The cases of special interest are the ones with parameters $a, b$ or $c$ of order $O\left(v^{2} / v_{\phi}^{2}\right)$ but $c \gg$ $\lambda_{i} v^{2} / v_{\phi}^{2}$ or $c \sim \lambda_{i} v^{2} / v_{\phi}^{2}$. For instance, in the last case $\rho$ is a mass eigenstate with mass $m_{h_{3}}^{2}=4 \lambda_{\phi} v_{\phi}^{2}$. The two remaining masses are

$$
m_{h_{1}, h_{2}}^{2}=8 v^{2}\left(K \mp \sqrt{K^{2}-L}\right),
$$

where $K=2\left(\lambda_{1} c_{\beta}^{2}+\lambda_{2} s_{\beta}^{2}+\lambda_{3}\right)+\frac{v_{\phi}^{2} c}{2 v^{2} s_{2 \beta}}, L=8\left[\left(\lambda_{1} \lambda_{2}+\lambda_{1} \lambda_{3}+\lambda_{2} \lambda_{3}\right) s_{2 \beta}^{2}+\frac{v_{\phi}^{2} c}{2 v^{2} s_{2 \beta}}\left(\lambda_{1} c_{\beta}^{4}+\lambda_{2} s_{\beta}^{4}+\lambda_{3}\right)\right]$.

These cases can provide much richer phenomenology (with $h_{2}$, charged Higgs and $\tilde{A}_{0}$ at the weak scale) than others not discussed here. One can notice that the presence of these light states requires that some couplings are rather small which my seem odd or fine-tuned. For a discussion on the 'naturalness' of this possibility see [7].

\section{Oblique corrections}

\subsection{Definitions and experimental measurements}

If the new physics scale is significantly higher than the electroweak scale, new physics effects from virtual particles in loops are expected to contribute predominantly through vacuum polarization corrections to the electroweak precision observables.

One can parametrize possible departures from the SM with the so-called oblique parameters. In our calculations we use the parameters $\epsilon_{1}, \epsilon_{2}$, and $\epsilon_{3}$ defined in [8] as follows:

$$
\epsilon_{1} \equiv \frac{1}{M_{W}^{2}}\left[A^{33}(0)-A^{11}(0)\right], \quad \epsilon_{2}=F^{11}\left(M_{W}^{2}\right)-F^{33}\left(M_{W}^{2}\right), \quad \epsilon_{3}=\frac{c}{s} F^{30}\left(M_{Z}^{2}\right) .
$$

$A^{i j}$ and $F^{i j}$ are the coefficients in the vacuum-polarization tensors

$$
\Pi_{\mu \nu}^{i j}(q)=-i g_{\mu \nu}\left[A^{i j}(0)+q^{2} F^{i j}\left(q^{2}\right)\right]+q_{\mu} q_{v} \text { terms, }
$$

where $i j$ may be either $W W, W_{3} W_{3}$ or $W_{3} B$ and $q_{\mu}$ is the four-momentum of the gauge boson. In the effective theory $\epsilon_{1}, \epsilon_{2}$, and $\epsilon_{3}$ receive one-loop contributions from the leading $O\left(p^{2}\right)$ term and the tree-level contributions from the $a_{i}(0)$ :

$$
\epsilon_{1}=2 a_{0}(0)+\ldots, \epsilon_{2}=-g^{2} a_{8}(0)+\ldots, \epsilon_{3}=-g^{2} a_{1}(0)+\ldots,
$$




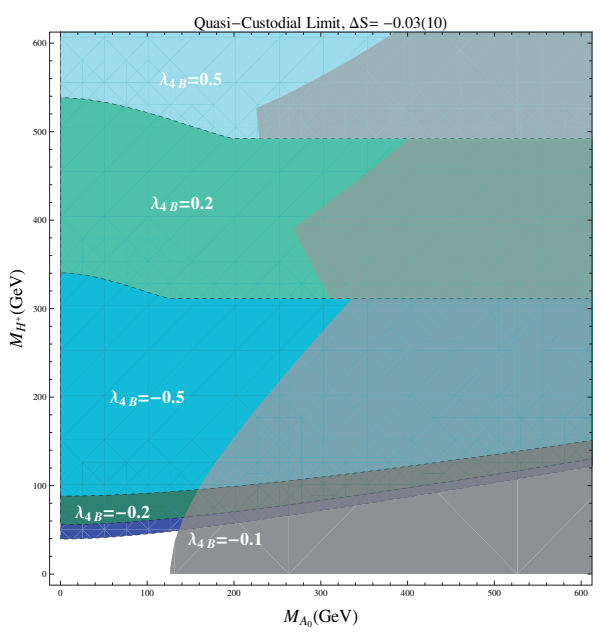

Figure 2. Exclusion plot imposed by the constraint from $\Delta S$ on the $\tilde{A}_{0}$ and $H_{ \pm}$in the 'quasi-custodial' limit for different values of the symmetry breaking parameter $\lambda_{4 B}$. Grey regions are excluded by stability.

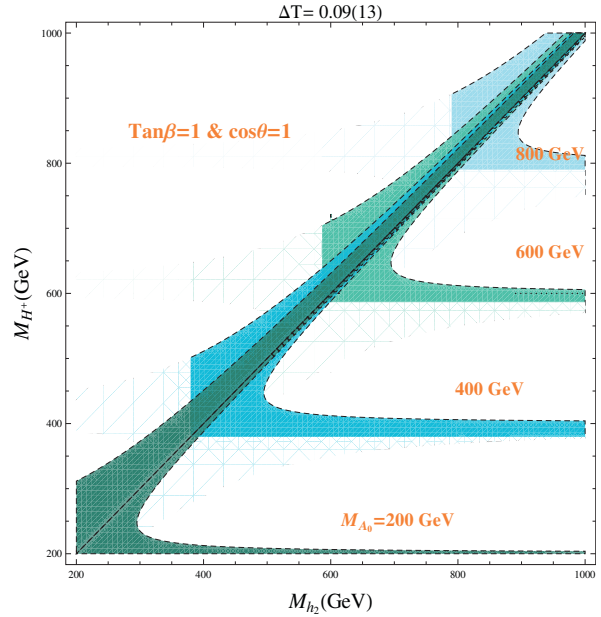

Figure 3. Exclusion plot imposed by the constraint from $\Delta T$ on the $h_{2}$ and $H_{ \pm}$. The successive horizontal bands correspond to different values of $m_{A_{0}}$. The stability bounds are implemented.

where the ellipses symbolize the one-loop $\frac{v^{2}}{4} \operatorname{Tr} D_{\mu} \mathcal{U}^{\dagger} D^{\mu} \mathcal{U}$ contributions.

The Feynman diagrams which contribute to the vacuum polarization in the DFSZ model are depicted in Fig. 1. The wavy lines correspond to gauge bosons and the usual ones to various scalar fields. Considering the gauge invariant kinetic term $\mathcal{L}_{\text {kin }}=\frac{1}{2}\left(\partial_{\mu} \phi\right)^{*} \partial^{\mu} \phi+\frac{1}{4} \operatorname{Tr}\left[\left(D_{\mu} \Phi_{12}^{\dagger}\right) D^{\mu} \Phi_{12}\right]$, one can get all types of interactions and consequently all contributions to $\epsilon_{i}$.

Experimental constraints on the oblique parameters are obtained from the global fits of the electroweak sector of the SM. For a long time, such fits have been used to exploit measurements of electroweak precision observables at lepton colliders (LEP, SLC), together with measurements at hadron colliders (Tevatron, LHC), and accurate theoretical predictions at multi-loop level, to constrain free parameters of the SM, such as the Higgs and top masses. Today, all fundamental SM parameters entering these fits are experimentally determined, including information on the Higgs couplings, and the global fits are used as powerful tools to assess the validity of the theory and to constrain scenarios for new physics.

While performing global fits( [9],[10]), it is more common to work with another set of parameters $S, T$ and $U$ determined firstly in [11]. The connection between $S, T$ and $U$, defined relative to the $\mathrm{SM}\left(\Delta T=T-T^{S M}, e t c.\right)$, and $\epsilon_{i}$ is as follows:

$$
\Delta T=\frac{\epsilon_{1}-\epsilon_{1}^{S M}}{\alpha}, \Delta U=-\frac{4 s_{W}^{2}\left(\epsilon_{2}-\epsilon_{2}^{S M}\right)}{\alpha}, \Delta S=\frac{4 s_{W}^{2}\left(\epsilon_{3}-\epsilon_{3}^{S M}\right)}{\alpha} .
$$

Here, $\alpha=e^{2} /(4 \pi)=g^{2} s_{W}^{2} /(4 \pi)$ is the fine-structure constant, $s_{W}=\sin \theta_{W}$ is the sine of the weak mixing angle $\theta_{W}$. 


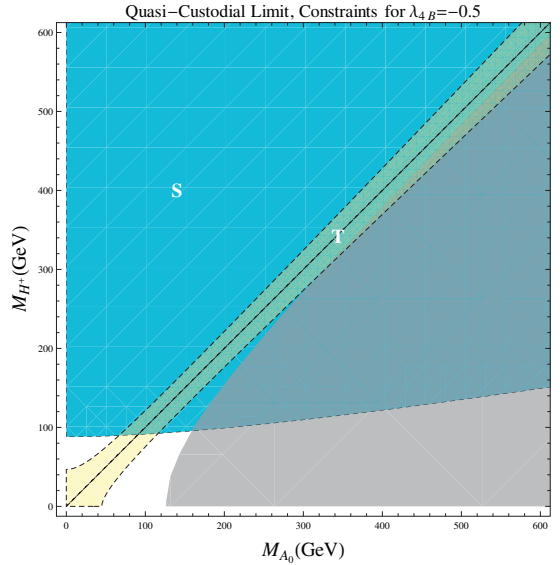

Figure 4. Restrictions both from $\Delta S$ and $\Delta T$ in the 'quasi-custodial' limit for $\lambda_{4 B}=-0.5$. Grey regions are excluded by stability.

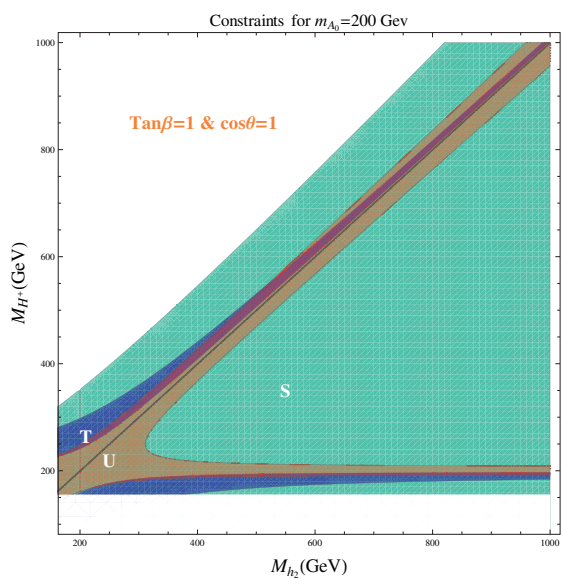

Figure 5. Restrictions from $\Delta S, \Delta T$ and $\Delta U \simeq 0$ on the mass spectrum. The stability bounds are implemented.

The experimental determinations of $\Delta S, \Delta T$ and $\Delta U$ are:

from the Gfitter group [9]: $\Delta S=0.05 \pm 0.11, \quad \Delta T=0.09 \pm 0.13, \quad \Delta U=0.01 \pm 0.11$;

from the PD paper [10]: $\Delta S=-0.03 \pm 0.10, \quad \Delta T=0.01 \pm 0.12, \quad \Delta U=0.05 \pm 0.10$.

$\Delta U$ is considered to be very small in most new physics models and therefore often set to zero in the global fits. This changes the experimental limits on $\Delta S, \Delta T$ a bit due to correlations between the parameters. The relevant constraints in the $\Delta U=0$ scenario are [9]:

$$
\left.\Delta S\right|_{\Delta U=0}=0.06 \pm 0.09,\left.\quad \Delta T\right|_{\Delta U=0}=0.1 \pm 0.07
$$

\subsection{Spectrum implications}

With both theoretical expressions for the oblique parameters and experimental bounds on them, we can explore the allowed range of masses. As we are basically interested in the possibility of obtaining a lightish spectrum, we discuss the case where the $c$ parameter scales as $v^{2} / v_{\phi}^{2}$.

We will assume two types of settings. First, a 'quasi-custodial' one, which means that the custodial symmetry is broken only via the coupling $\lambda_{4 B}=\lambda_{4}-2 \lambda$ being non-zero. The rotation matrix of $0^{+}$ states is equal to unity. The mass spectrum gets an additional restriction: $m_{H_{ \pm}}^{2}=m_{h_{2}}^{2}+8 v^{2} \lambda_{4 B}$. In Fig. 2 we provide an exclusion plot from the constraints on $\Delta S$ as an example. Notice, that the negative values of $\lambda_{4 B}$ allow much lighter spectra than the positive ones.

Then, the general setting, when the three masses $m_{\tilde{A}_{0}}, m_{H_{ \pm}}$and $m_{h_{2}}$ are unrelated, except for the eventual lack of stability of the potential. The rotation matrix can be different form the identity. However, experimentally the parameter $\cos \theta$, appearing on the diagonal of the rotation matrix, is known [6] to be very close to one. We present a range of masses allowed by the constraints on $\Delta T$ in Fig. 3. 
Furthermore, we depict constraints from several oblique parameters simultaneously. For the particular choice of $\lambda_{4 B}$ in the 'quasi-custodial' setting we have the exclusion plot both from $\Delta S$ and $\Delta T$ in Fig. 4. $\Delta T$ significantly narrows the allowed region, while $\Delta U$ gives nothing new and is not present in the plot. For $\lambda_{4 B}=0.2$ the possible range would start at about $1 \mathrm{TeV}$ range, while for negative $\lambda_{4 B}$ it goes down to $100 \mathrm{GeV}$. If we completely give up the custodial symmetry, $\Delta T$ determines the most severe restrictions on the spectrum generally. However, $\Delta U \simeq 0$ scenario brings even more restrictions, see Fig. 5. As $\Delta U$ gets closer to exact zero its region shrinks to two lines, which overlap with $\Delta T$ region only asymptotically at infinity.

\section{Conclusions}

The nature of electroweak symmetry breaking keeps being an important issue in particle physics today. The Standard Model of particle physics contains a mechanism for electroweak symmetry breaking, and the discovery of the Higgs boson at the LHC proves its consistency. However, the Minimal Standard Model still has a several well-known problems. One of them being the absence of the degree of freedom for dark matter.

The Dine-Fischler-Srednicki-Zhitnitsky model is a model with the electroweak symmetry breaking pattern, similar to the SM. The model contains an invisible axion which is an interesting candidate for dark matter. Being extremely weakly coupled, the axion cannot be directly detected at the LHC. Hence, we make an investigation what indirect consequences of the axion presence can be seen experimentally.

We consider the constraints from electroweak precision parameters, expressed in terms of the oblique parameters, to get the restrictions on the model spectrum. The large scale, appearing in the DFSZ model to make the axion nearly invisible, seems to generate a very heavy and inaccessible spectrum of the new physics. However, we discuss cases in which a rather light spectrum appears, with even a possibility to be tested at the LHC.

\section{References}

[1] R. D. Peccei and H. R. Quinn, Phys. Rev. Lett. 38 (1977) 1440; Phys. Rev. D16 (1977) 1791.

[2] M. Dine, W. Fischler and M. Srednicki, Phys. Lett. B 104, 199 (1981).

[3] A.R. Zhitnitsky, Sov. J. Nucl. Phys. 31, 260 (1980) [Yad. Fiz. 31, 497 (1980)].

[4] P. Ciafaloni and D. Espriu, Phys. Rev. D 56, 1752 (1997) [hep-ph/9612383].

[5] R.L. Delgado, A. Dobado and F. J. Llanes-Estrada, JHEP 1402, 121 (2014); D. Espriu, F. Mescia and B. Yencho, Phys. Rev. D 88, 055002 (2013) [arXiv:1307.2400 [hep-ph]].

[6] D. Espriu, F. Mescia and A. Renau, arXiv:1503.0295 [hep-ph].

[7] R. R. Volkas, A. J. Davies and G. C. Joshi, Phys. Lett. B 215, 133 (1988); R. Foot, A. Kobakhidze, K. L. McDonald and R. R. Volkas, Phys. Rev. D 89, no. 11, 115018 (2014) [arXiv:1310.0223 [hep$\mathrm{ph}]]$.

[8] G. Altarelli and R. Barbieri, Phys. Lett. B 253, 161 (1990).

[9] M. Baak, J. Cuth, J. Hallec, A. Hoecker, R. Kogler, K. Monig, M. Schott, J. Stelzer, Eur. Phys. J. C 74, 3046 (2014) [arXiv:1407.3792v1 [hep-ph]].

[10] K.A. Olive et al. (Particle Data Group), Chin. Phys. C 38, 090001 (2014).

[11] M. Peskin and T. Takeuchi, Phys. Rev. Lett. 65, 1964 (1990). 